\title{
Whole transcriptome profiling of patient-derived xenograft models as a tool to identify both tumor and stromal specific biomarkers
}

\author{
James R. Bradford ${ }^{1}$, Mark Wappett ${ }^{2}$, Garry Beran ${ }^{2}$, Armelle Logie ${ }^{2}$, Oona Delpuech ${ }^{2}$, \\ Henry Brown'2, Joanna Boros', Nicola J. Camp', Robert McEwen², Anne Marie \\ Mazzola ${ }^{4}$, Celina D'Cruz ${ }^{4}$, Simon T. Barry ${ }^{2}$ \\ ${ }^{1}$ Department of Oncology and Metabolism, University of Sheffield, Sheffield, South Yorkshire, UK \\ ${ }^{2}$ Oncology iMED, AstraZeneca Pharmaceuticals, Alderley Park, Cheshire, UK \\ ${ }^{3}$ Department of Internal Medicine and Huntsman Cancer Institute, University of Utah, Salt Lake City, Utah, USA \\ ${ }^{4}$ Oncology iMED, AstraZeneca Pharmaceuticals, Gatehouse Park, Massachusetts, USA \\ Correspondence to: James R. Bradford, e-mail: J.R.Bradford@sheffield.ac.uk
}

Keywords: patient-derived xenograft, RNA-Seq, tumor stroma, biomarker discovery, pre-clinical research

Received: November 09, 2015

Accepted: February 18, 2016

Published: March 09, 2016

\section{ABSTRACT}

The tumor microenvironment is emerging as a key regulator of cancer growth and progression, however the exact mechanisms of interaction with the tumor are poorly understood. Whilst the majority of genomic profiling efforts thus far have focused on the tumor, here we investigate RNA-Seq as a hypothesis-free tool to generate independent tumor and stromal biomarkers, and explore tumor-stroma interactions by exploiting the human-murine compartment specificity of patient-derived xenografts (PDX).

Across a pan-cancer cohort of 79 PDX models, we determine that mouse stroma can be separated into distinct clusters, each corresponding to a specific stromal cell type. This implies heterogeneous recruitment of mouse stroma to the xenograft independent of tumor type. We then generate cross-species expression networks to recapitulate a known association between tumor epithelial cells and fibroblast activation, and propose a potentially novel relationship between two hypoxiaassociated genes, human MIF and mouse Ddx6. Assessment of disease subtype also reveals MMP12 as a putative stromal marker of triple-negative breast cancer. Finally, we establish that our ability to dissect recruited stroma from trans-differentiated tumor cells is crucial to identifying stem-like poor-prognosis signatures in the tumor compartment.

In conclusion, RNA-Seq is a powerful, cost-effective solution to global analysis of human tumor and mouse stroma simultaneously, providing new insights into mouse stromal heterogeneity and compartment-specific disease markers that are otherwise overlooked by alternative technologies. The study represents the first comprehensive analysis of its kind across multiple PDX models, and supports adoption of the approach in pre-clinical drug efficacy studies, and compartment-specific biomarker discovery.

\section{INTRODUCTION}

The tumor stroma comprises of numerous cell types including endothelial cells, cancer-associated fibroblasts (CAFs), mesenchymal stem cells, and immune cells such as lymphocytes and tumor-associated macrophages. It plays a critical role in supporting cancer growth and metastasis [1], and is therefore emerging as rich source of targets for anti-cancer therapy. However, we have a poor understanding of the interactions between tumor and stroma. A typical solid tumor tissue sample consists of both components, and such sample heterogeneity can have significant influence on the biological interpretation of genomic profiling studies [2]. Furthermore, attempts 
to separate tumor from stroma are hampered by the requirement for specialist techniques such as laser capture micro-dissection, with small amounts of tumor cell contamination possible.

Patient-derived tumor xenograft (PDX) models are generated when fresh tumor tissue obtained directly from patients is implanted subcutaneously or orthotopically into immune-deficient mice. As such, they maintain the principal histological, clinical and molecular characteristics of the original patients' tumors while remaining biologically stable when passaged in mice [3-5]. Since PDX models more closely resemble and recapitulate tumor growth in humans than standard in vitro cell line or cell line xenograft approaches, they remain key experimental platforms for pre-clinical drug development.

Recent studies have shown that human and mouse transcription can be accurately differentiated in PDX models using RNA-Seq [6-7], removing the need for manipulation of the RNA population, customised sequencing protocols, or prior knowledge of the species component ratio. Moreover, the known transcriptional response to drugs targeting the stroma can be accurately recapitulated in both human tumor and mouse stroma [6]. The high specificity of the in silico read disambiguation approach means that gene expression in the human component is quantified almost exclusively from tumor RNA, particularly in later passages where the original patient stroma has been replaced by mouse stroma. Thus PDX transcriptome data provide a unique opportunity for the simultaneous study of both tumor and stromal specific signals in vivo. Consequently, several studies have adopted the approach albeit restricted to only a small number of xenograft models or specific cancer type [8-9].

In this paper, we build significantly on these early studies by using RNA-Seq to profile the baseline human and mouse transcriptome of 79 PDX models representing multiple cancer types. By doing so, we assess mouse stromal heterogeneity, generate hypotheses on the relationship between mouse stroma and human tumor, and identify both tumor and stromal specific markers of disease subtype. To our knowledge, this represents the first comprehensive analysis of both species components of PDX models simultaneously across such a large cohort. As such, the dataset should provide a key platform for gaining additional insights into PDX tumor and stroma processes, and interpreting pre-clinical efficacy studies.

\section{RESULTS}

79 PDX models from five different providers covering seven cancer types were used in the study (Figure 1A, Table S1) with the majority of models representing lung (37 models) and breast (19 models). A mean of 47,906,117 human and 6,612,995 mouse reads were uniquely mapped to a concatenated human and mouse genome (Figure S1A, Table S2), corresponding to a human component of $\sim 88 \%$ in each sample (Figure S1B, Table S2). We have previously demonstrated that low coverage (1.3-2.0 M reads) was sufficient to deliver accurate detection and measurement of mouse gene expression [6]. In this study we generated a coverage of 5-10 $\mathrm{M}$ reads, which was considered sufficient to generate a robust data set. Expression between technical and biological replicates showed strong correlation across both human (Figure S2A-S2C) and mouse (Figure S2D-S2F) genes. Predicted stromal and immune cell content using ESTIMATE [10] indicated a clear differentiation between the human and mouse components, and $>97 \%$ tumor purity in the human component of 76/79 samples (Figure S3, Table S3). As a proxy for the quantity of original patient stroma in each PDX sample, human expression levels of two CAF markers that are rarely expressed by tumor epithelial cells, fibroblast activation protein alpha $(F A P)$ and chondroitin sulfate proteoglycan 4 (CSPG4), were assessed. 22/79 samples showed evidence of patient stroma retention at a low stringency CAF marker expression threshold (FAP or CSPG4 $\log _{2}$ FPKM > 2.0; Table S3) and therefore flagged as potential confounders in analyses of the human component. Of these, only 11 samples expressed high levels of either marker $\left(\log _{2}\right.$ FPKM > 4.0), and overall results suggest human and mouse transcriptional profiles reflect highly enriched human tumor and mouse stroma cell populations respectively in the majority of samples.

\section{Mouse stroma heterogeneity is primarily driven by dominant cell type}

We applied non-negative matrix factorization (NMF) to cluster 14173 and 3933 of the most highly expressed (human: FPKM $>10$, mouse: FPKM $>2$ in at least one sample) and variable (coefficient of variation $>0.20$ ) genes across human and mouse respectively, and test whether gene expression signatures exist in the mouse component allowing separation into distinct subtypes. Stable clusters were achieved at $k=9$ (human; Figure 1B) and $k=5$ (mouse; Figure 1D) where $k$ denotes the number of clusters and values selected according to the procedure outlined in Methods. Model-to-cluster mappings for both human and mouse are given in Tables S4A and S4B respectively, and genes deemed as key drivers of the clustering (meta-genes) are listed in Tables S5A (human) and S5B (mouse).

Human clusters were more strongly associated with cancer type ( $p<2.20 \mathrm{E}-16$ by Chi-squared test; Figure 1C) than the mouse clusters ( $p=1.07 \mathrm{E}-5$; Figure 1E). 8/15 and $7 / 11$ tumors in human clusters 1 and 2 respectively expressed CAF markers FAP/CSPG4, which may suggest a propensity for some lung squamous and triple-negative breast cancers (TNBC) to retain patient stroma.

Functional enrichment analysis using Toppgene [11] revealed that $3 / 5$ mouse clusters encompassed strong functional themes associated with fat (cluster 1), muscle (cluster 3) and immune cells (cluster 5; Figure 1F, Table S6) corresponding to strong overlap with adipocyte $(p=1.70 \mathrm{E}-16)$, embryonic stem $(p=2.14 \mathrm{E}-37)$, and 
myeloid ( $p=8.68 \mathrm{E}-28$ ) cell type signatures respectively. Cluster 2 was primarily driven by Col10a1 expression (relative contribution to meta-gene $=0.89$; Table S5B), a potential marker of CD10+ tumor stromal cells [12], and cluster 4 showed some enrichment for mesenchymal stem cell markers ( $p=1.01 \mathrm{E}-08)$.

The mean number of mapped mouse reads (Figure S4A) or proportion of mouse component (Figure S4B) was not significantly different between mouse clusters. Notably, cluster 5 included samples from model HOXF060 with the largest mouse component in the cohort $(69.8 \%$; $35,614,081$ mapped reads), and HPAXF049 with one of the lowest mouse components $(7.5 \% ; 3,109,853$ mapped reads). Despite the difference in coverage, high mouse gene expression correlation was achieved between these samples $(r=0.93$; Figure S5), suggesting coverage was not a major confounding factor. Furthermore, no significant association was observed between the mouse clusters and mouse gender $(p=0.10)$ or tumor stage (primary or metastatic; $p=0.55)$, and only some association with mouse strain $(p=3.09 \mathrm{E}-04)$. This was possibly driven by membership of all athymic nude mice in clusters 1 and 4 (Figure 1E), otherwise, it was difficult to discern possible influence of mouse strain on dominant stromal cell type from these data alone.

\section{Inferring tumor-stroma crosstalk from inter- species gene expression correlations}

Given the potential for heterogeneous recruitment of mouse stroma to the human tumor, we next performed a comprehensive expression correlation analysis between human and mouse components to generate hypotheses on tumor-stroma crosstalk. We initially looked for evidence of a known tumor-stroma association, selecting the recent observation that fibroblast activation is associated with a tumor epithelial cell type [13]. Expression of human epithelial cadherin $(C D H 1)$ and epithelial cell adhesion molecule (EPCAM) were used as epithelial markers, and mouse expression of fibroblast associated protein (Fap), chondroitin sulfate proteoglycan 4 (Cspg4) and alphasmooth muscle actin (Acta2) as CAF markers. In five of the six possible cross-species comparisons, a significant Pearson correlation coefficient $(p<0.05)$ was observed between the human epithelial and mouse CAF markers (Table 1; Figure S6A-S6F), particularly between EPCAM and Fap $(r=0.37, p=7.90 \mathrm{E}-4$; Figure S6A) indicating the positive relationship between tumor epithelial cell type and fibroblast activation exists in the PDX panel. No significant correlation was observed between the mouse CAF markers
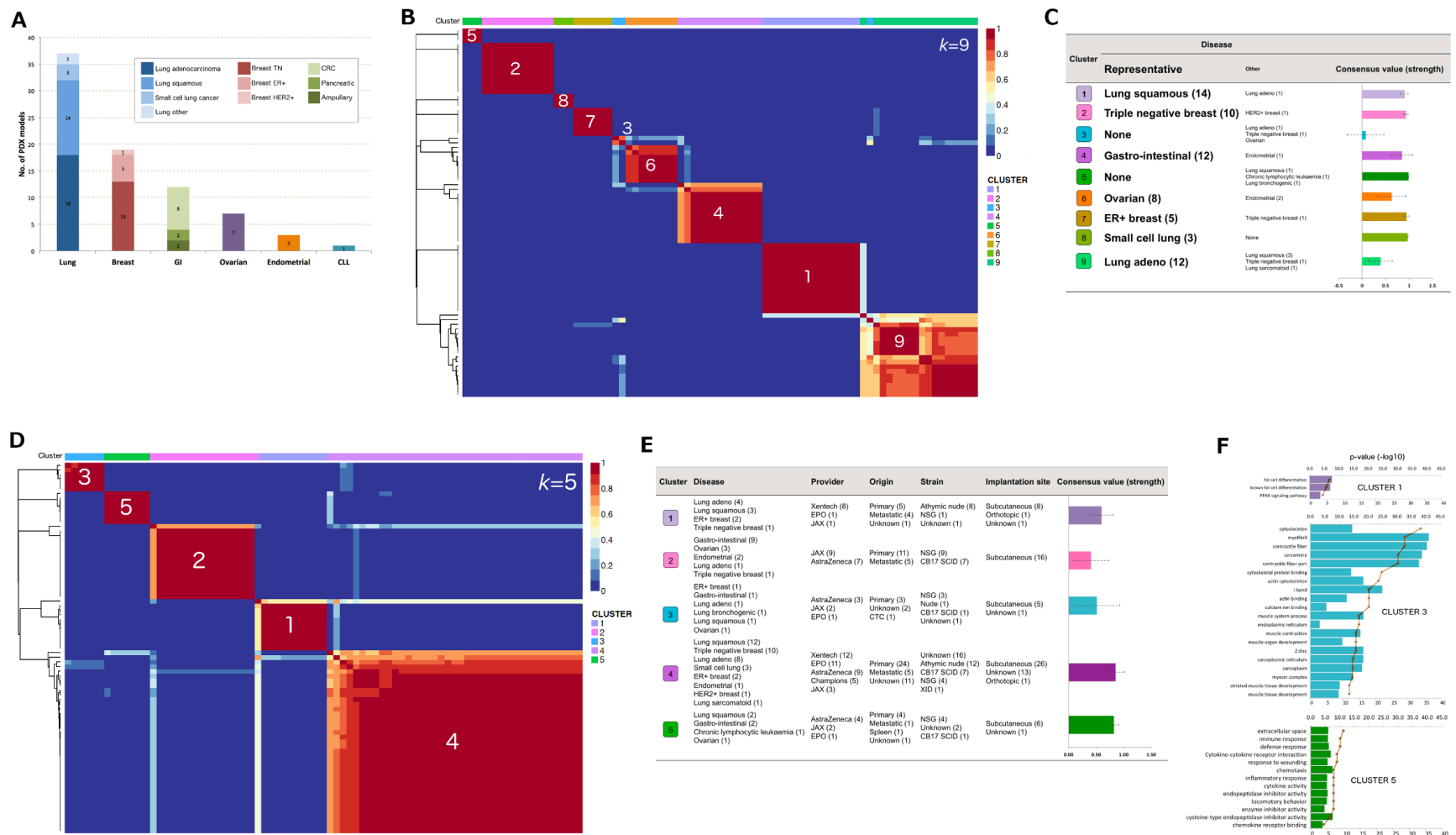

$\mathbf{E}$

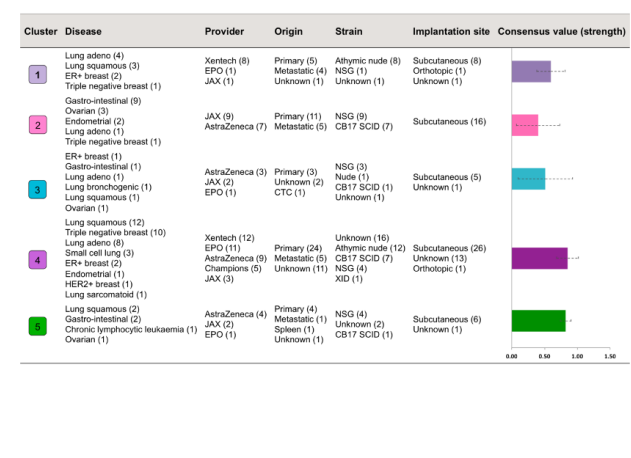

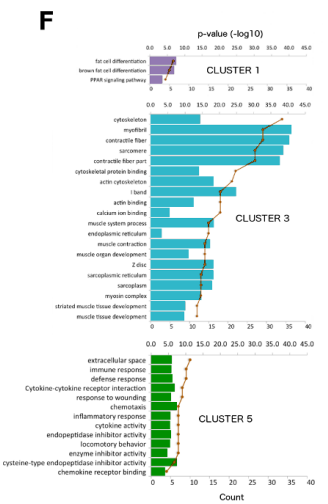

Figure 1: Application of non-negative matrix factorization (NMF) to optimal clustering of human and mouse gene expression. (A) disease representation across the 79 PDX models. (B) consensus matrix at $k=9$ for the human transcriptome. (C) contributing cancer types and mean consensus value of each human cluster. "Representative" disease indicates the majority cancer type in the cluster, and numbers of models are given in brackets. Mean consensus value was computed from 200 runs of NMF. (D) consensus matrix at $k=9$ for the mouse transcriptome. (E) meta-data breakdown in each mouse cluster and mean consensus value. (F) functional enrichment of the meta-genes driving the mouse clustering. Only clusters with significant functional enrichment $($ FDR $<0.05)$ are shown. 
Table 1: Pearson correlation coefficients calculated between human epithelial and mouse CAF markers

\begin{tabular}{|l|c|c|c|}
\hline \multirow{2}{*}{ Human genes } & \multicolumn{3}{c}{ Mouse genes } \\
\cline { 2 - 4 } \multicolumn{1}{c|}{ Acta2 } & Fap & Cspg4 \\
\hline CDH1 & $0.29^{* *}$ & $0.24^{*}$ & $0.32^{* *}$ \\
\hline EPCAM & 0.06 & $0.37^{* *}$ & $0.27^{*}$ \\
\hline VIM & 0.01 & -0.20 & 0.10 \\
\hline$Z E B 1$ & -0.01 & -0.21 & -0.05 \\
\hline$* * p<0.01, * p<0.05$ & & & \\
\hline
\end{tabular}

and two human mesenchymal cell type markers vimentin (VIM) and zinc finger E-box binding homeobox 1 (ZEB1; Table 1), with Fap achieving weak anti-correlation with both VIM $(r=-0.20, p=0.08)$ and ZEB1 $(r=-0.21$, $p=0.06$ ). Within the context of all correlations between human $\mathrm{CDH1}$ and 2495 most highly expressed and variable mouse genes, both Fap and Cspg4 were ranked in the top $6 \%$ most correlated genes (Table S7). Notably, a significant number of these top $6 \%$ genes play a role in cell migration $(p=8.37 \mathrm{E}-13)$ and vasculature development $(p=3.12 \mathrm{E}-10)$, both characteristic of an activated stroma, including the top ranked gene collagen, type VIII, alpha 1 (Colsa1, $r=0.67$; Figure S7), a key component of blood vessel endothelia.

To explore potentially novel gene-wise associations between human tumor and mouse stroma, an all-againstall comparison of 14,336 expression profiles representing the most highly expressed and variable genes from both the human (11,841 genes) and mouse (2495) components was performed. The resulting co-expression network consisted of 259,627 edges and 7089 nodes (Figure 2A) where nodes represented genes and edges were drawn between gene pairs achieving a stringent threshold of $|r|>0.85$ $(p<2.20 \mathrm{E}-16)$ to control for false positives. The majority of edges $(256,922)$ were between positively correlated human gene pairs, with the remainder of the network comprising of 2661 edges between mouse gene pairs, and 44 cross-species anti-correlated gene pairs. The strongest cross-species anti-correlation $(r=-0.90)$ was observed between human macrophage migration inhibitory factor $(M I F)$ and mouse DEAD-box RNA helicase 6 ( $D d x 6$ ) (Figure 2B). In addition, human MIF (connected to 17 mouse genes) and mouse $D d x 6$ (connected to 8 human genes) represented the most connected cross-species nodes in the network (Table S8).

Increased MIF expression and reduced DDX6 expression are both known drivers of angiogenesis and strongly associated with VEGF activity, as such they have been implicated in tumor response to hypoxia [14-15]. Consistent with their known roles, positively correlated first or second neighbors of $M I F$ in the network (Figure 2A) were enriched for mitochondrial processes $(p=7.15 \mathrm{E}-05)$ and oxidative phosphorylation ( $p=1.72 \mathrm{E}-04)$, whereas mouse genes neighboring $D d x 6$ were enriched for other RNA binding genes $(p=3.93 \mathrm{E}-08)$. To test the association with hypoxia more explicitly, models were divided into MIF high/ Ddx6 low $\left(\log _{2}\right.$ FPKM human MIF $>11.5$, mouse $D d x 6$ $<1.05)$ and MIF low/Ddx6 high $(M I F<7.0, D d x 6>1.8)$ corresponding to possible hypoxic and normoxic samples respectively (Table S9). Genes differentially expressed $\left(\log _{2}\right.$ fold change $(\mathrm{FC})>1.50$, false discovery rate $(\mathrm{FDR})<0.05)$ between the two groups were then identified. According to Toppgene, genes over-expressed in $M I F$ high/Ddx6 low samples were enriched $(p=1.50 \mathrm{E}-06)$ for signatures representing genes up-regulated under hypoxia in vivo [16]. Conversely, genes over-expressed in $M I F$ low/Ddx6 high samples were enriched $(p=5.09 \mathrm{E}-05)$ for genes downregulated in the same study, thus supporting the association of high human $M I F$ and low mouse $D d x 6$ expression with tumor hypoxia.

Closer inspection of all possible inter- and intraspecies correlations between MIF and DDX6 (Figure 2C) revealed no significant association $(p<0.01)$ between human DDX6 and either human MIF $(r=0.23, p=0.04)$, mouse $D d x 6$ ( $r=-0.04, p=0.75)$, or mouse $\operatorname{Mif}(r=-0.17$, $p=0.14)$. By contrast, human MIF and mouse Mif achieved the second highest correlation $(r=0.72)$ between human and mouse orthologs, suggesting that MIF has complementary roles in the tumor and mouse stroma.

\section{PDX models as a source of tumor and stroma specific markers of disease subtype}

We next focused on lung and breast cancer as the two most highly represented diseases in the PDX cohort, in order to assess the potential of our approach to identify clinically relevant, independent human tumor and mouse stroma markers of disease subtype. 


\section{Stromal-specific markers of lung and breast cancer subtype}

Comparison between mouse components of 14 lung adenocarcinomas and 18 squamous carcinomas identified no significantly differentially expressed genes, suggesting uniform recruitment of mouse stroma to human lung xenografts. By contrast, in the breast cancer panel, 18 mouse genes achieved significant fold change (|log2 $\mathrm{FC} \mid>1.5, \mathrm{FDR}<0.05$ ) between four ER+ luminal-B and nine basal-like TNBCs (BTNBC; Table S10). Such a low number of genes is in broad agreement with a dataset of patient tumor stroma samples isolated by laser capture micro-dissection (Finak_Stroma) [17] in which only 29 genes were differentially expressed $(|\log 2 \mathrm{FC}|>1.5$, FDR $<0.05$; Table S12). However, there was little overlap between the two gene lists with matrix metallopeptidase 12 (Mmp12) the only gene over-expressed in both mouse $\left(\log _{2} \mathrm{FC}=2.00, \mathrm{FDR}=2.43 \mathrm{E}-02\right)$ and clinical $\left(\log _{2} \mathrm{FC}\right.$ $=2.83, \mathrm{FDR}=3.14 \mathrm{E}-09)$ stroma. Interestingly, whilst MMP12 expression was largely absent in the PDX human component (mean $\log _{2} \mathrm{FPKM}=0.44$ ) and cell lines of the Cancer Cell Line Encyclopaedia [18] (CCLE; mean $\log _{2}$ signal $=4.51)$ representing pure tumor cell populations
(Figure 3A), significant over-expression in BTNBC was detected in clinical samples from The Cancer Genome Atlas [19] (TCGA; $\left.\log _{2} \mathrm{FC}=2.89, \mathrm{FDR}=2.67 \mathrm{E}-14\right)$ and the Utah Breast Cancer Study (UBCS; $\log _{2} \mathrm{FC}=2.56, \mathrm{FDR}=$ $1.21 \mathrm{E}-02$; Figure $3 \mathrm{~B}$ ). Thus significant fold changes appear restricted to only those samples containing recruited stroma (PDX mouse component, Finak_Stroma, and clinical tumor samples), suggesting that $M M P 12$ expression is specific to BTNBC stroma, and absent in tumor cells.

\section{Tumor-specific markers of BTNBC}

Exploiting the tumor purity of the PDX human component, we derived a putative set of tumor-specific BTNBC markers by comparing a list of 1127 genes overexpressed $\left(\log _{2} \mathrm{FC}>1.5\right.$, FDR $\left.<0.05\right)$ in BTNBC from the PDX human component with 273 genes over-expressed $\left(\log _{2} \mathrm{FC}>1.5, p<0.001\right)$ in cell lines. This resulted in an overlap of 117 genes on platforms representing high tumor content (Figure 3A). We then performed a second overlap between this tumor-specific gene signature and 6156 differentially expressed genes detected in TCGA and UBCS at FDR $<0.05$ but ignoring fold change magnitude. By doing so, we reasoned that genes unique
A

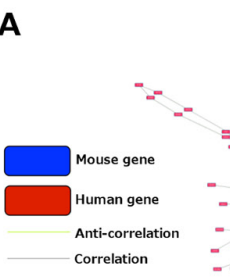

Full network

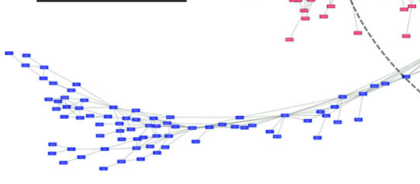

B

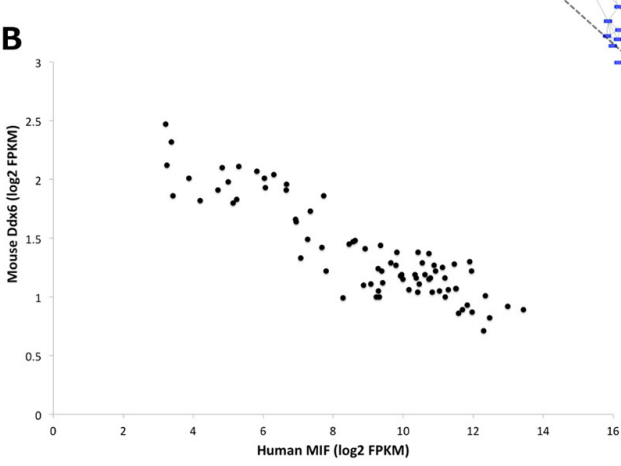

C

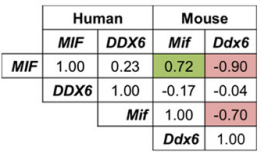

Network of first and second neighbours of MIF and Ddx6

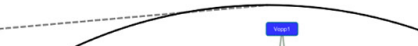


to the intersection between PDX and cell line datasets represented BTNBC markers that could only be observed in samples with high tumor purity, and overlooked in clinical samples containing a mixture of tumor cells and recruited stroma. Five genes fulfilled the criteria (Figure 3C): transmembrane glycoprotein NMB (GPNMB), CD44, tolllike receptor 3 (TLR3), anthrax toxin receptor 1 (ANTXR1) and lysyl oxidase $(L O X)$. All five genes retained significant differential expression after removal of five breast cancer samples (HBCX10, HBCX11, HBCX24, HBCX6 and HBCX9) expressing CAF markers FAP/CSPG4 in the human component, addressing the possibility that persistent retention of patient stroma in the BTNBC PDX models was confounding results.

To reproduce the tumor purity of the PDX human component and cell lines in clinical samples, we performed a second comparison between 12 BTNBC and 10 ER+ luminal-B TCGA patients representing samples with the highest estimated tumor cell content (ESTIMATE stromal score $<-700$ ). Remarkably, significant differential expression emerged in GPNMB $\left(\log _{2} \mathrm{FC}=1.19\right.$, FDR $=$ $1.18 \mathrm{E}-02)$ and $L O X\left(\log _{2} \mathrm{FC}=2.07, \mathrm{FDR}=2.53 \mathrm{E}-05\right)$ with the fold change of a third gene, ANTXR1 also increasing but not to significance $\left(\log _{2} \mathrm{FC}=0.74, \mathrm{FDR}=0.13\right.$; Figure $\left.3 \mathrm{D}\right)$. This suggests that at least two of the five genes are BTNBC markers expressed in patient samples, whose significance is masked by the difficulty of separating recruited stroma from tumor cells of stromal phenotype.

\section{Tumor-specific BTNBC markers are enriched for genes associated with a stromal phenotype}

To gain further insight into potential BTNBC markers derived from high tumor content samples, we next compared 1127 and 1368 genes over-expressed $\left(\log _{2} \mathrm{FC}>1.5\right.$, FDR $<0.05)$ in BTNBC from the PDX human component and TCGA patient samples respectively. This resulted in an overlap of 574 genes.

We initially speculated that the differences between the lists could in part be attributed to the presence of recruited stroma in typical clinical samples that is absent in the PDX human component (Figure 3A), so we included a 137-stromal gene signature [10] (Table S13A) in the comparison. Surprisingly, we observed greater overlap ( $p=6.00 \mathrm{E}-04$ by hyper-geometric test) of stromal signature genes with BTNBC markers derived from the PDX human component than those from clinical samples $(p=1.00$; Table 2; Figure 3E; Table S14). To understand whether this overlap was indicative of specific cell type, we also overlaid epithelial-mesenchymal transition [20] (EMT; Table S13B), breast cancer stem cell [21] (CSC; Table S13C) and reactive stromal [22] (Table S13D) signatures. Clear enrichment ( $p=7.77 \mathrm{E}-16)$ of mesenchymal markers including VIM was observed in the BTNBC PDX human component. By contrast, only a slight enrichment was observed $(p=0.07)$ in clinical BTNBC samples and whilst $V I M$ achieved significant fold change, it was small $\left(\log _{2} \mathrm{FC}=1.13, \mathrm{FDR}=2.15 \mathrm{E}-15\right)$ compared to that observed from the PDX human component $\left(\log _{2} \mathrm{FC}=4.49\right.$, $\mathrm{FDR}=2.18 \mathrm{E}-05)$. There was no enrichment of stromal or mesenchymal cell markers in genes over-expressed across either PDX or clincal ER+ luminal-B samples (Table 2; Table S14). The BTNBC PDX human component was also enriched for reactive stroma $(p=6.08 \mathrm{E}-04)$ and breast CSC markers ( $p=1.60 \mathrm{E}-04$; Table 2; Table S14), and whilst a high number of non-CSC markers were also present, the enrichment was not significant ( $p=0.53$ ). Furthermore, CD44, a marker for cells with tumor initiating potential, was identified as a BTNBC marker in both PDX $\left(\log _{2} \mathrm{FC}=2.14, \quad \mathrm{FDR}=5.91 \mathrm{E}-04\right)$ and cell line $\left(\log _{2} \mathrm{FC}=2.58, p=8.66 \mathrm{E}-03\right)$ but not in clinical $\left(\log _{2} \mathrm{FC}=0.06, \mathrm{FDR}=0.79\right)$ datasets. Low CD24 expression is also indicative of tumor initiating potential, however human CD24 expression was not detected across any of the PDX samples. Conversely, non-CSC markers were enriched in genes over-expressed in PDX ( $p=1.26 \mathrm{E}-03)$ and TCGA ER+ luminal-B samples ( $p=1.29 \mathrm{E}-05)$.

Encouragingly, BTNBC markers remained enriched for stromal ( $p=3.30 \mathrm{E}-03)$, EMT ( $p=4.17 \mathrm{E}-10)$, CSC $(p=1.59 \mathrm{E}-03)$ and reactive stromal $(p=3.04 \mathrm{E}-03)$ signatures (Figure 3F; Table 2; Table S14) after removal of the five breast PDX models expressing CAF markers $F A P$ and CSPG4 suggesting the presence of stromallike signature genes in the PDX human component was independent of patient stroma retention. Furthermore, there was no evidence of bias towards mesenchymal or mesenchymal stem-like (MSL) TNBC subtypes [23] (Table S11), indeed no MSL or immunomodulatory (IM) subtypes were present in the PDX data in accordance with the view that these subtypes are likely defined by high expression of genes from the micro-environment rather than the tumor itself [24]. Therefore our observations are unlikely due to over-representation of the stromal subtype in PDX BTNBC models.

\section{Stromal phenotype emerges in BTNBC clinical samples of high tumor content}

As before, we reproduced the tumor purity of the PDX human component in clinical samples by focusing on TCGA samples with the lowest predicted stromal cell content (ESTIMATE stromal score $<-700$ ). Remarkably, enrichment of both EMT ( $p=2.05 \mathrm{E}-07)$ and CSC $(p=1.83 \mathrm{E}-02)$ signatures emerged in genes differentially expressed between the remaining $12 \mathrm{BTNBC}$ and $10 \mathrm{ER}+$ luminal B TCGA samples, comparable to that observed in the PDX human component (Figure 4C). The numbers of stromal and reactive stromal signature genes also increased although not sufficient to achieve significance. Taken together, these findings support the presence of a subpopulation of cells with CSC and mesenchymal features in BTNBC that is difficult to detect in a typical clinical sample due to equivalent expression signals from the recruited stroma. 


\section{DISCUSSION}

We describe the output of a comprehensive expression analysis of 79 pan-cancer PDX models using an RNA-Seq generated species-specific mapping strategy. Accurate separation of human and mouse components of the tumor allows use of PDX tumors to gain unique insights into tumor-stroma crosstalk, and through cross comparison, generate both tumor and stroma expression signatures that give insights specific to disease subtypes, and also aid development of biomarker signatures. When passaged in vivo much of the patient stroma present upon implantation and early passages is eventually replaced by murine stroma, and whilst this can lead to loss of some original features found in the patient tumor microenvironment [25], it is key to enabling non-invasive species-specific separation of PDX tumor from stroma in silico. Therefore, it offers a unique opportunity not only to look at features specific to the stroma but also reveal new biology within the human tumor cells.

\section{Identification of distinct mouse stromal clusters defined by dominant cell type}

Mouse gene expression profile clustering revealed distinct PDX stromal subtypes broadly related to dominant cell types characterized by adipocyte, embryonic stem, and myeloid signatures, the latter indicating a high level of inflammatory infiltrate in the mouse stroma. These were independent of cancer type and PDX provider and suggest heterogeneous recruitment of mouse stroma to the human tumor. Since interaction between the tumor and stroma is known to influence drug response, our findings imply that the effect of the dominant stromal cell type in an individual model should be considered when analyzing therapeutic response. This is particularly pertinent for compounds that may drive efficacy through stromal elements, or be subject to stromal derived resistance. However, whilst the approach provides the opportunity to explore the association of stromal subtype and the degree of inflammatory infiltrate with survival outcome and drug resistance, it is important to characterize the models carefully. In this study of commonly used models there was a notable enrichment of high-grade tumors with poor prognosis [26], which will influence the outcome of such studies.

\section{Human-mouse gene correlation analysis identifies both known and potentially novel tumor-stroma associations}

The degree of transcriptional heterogeneity in the mouse component, and the potential for active involvement of the xenograft in the process prompted us to look more closely at gene expression correlations between the human
A

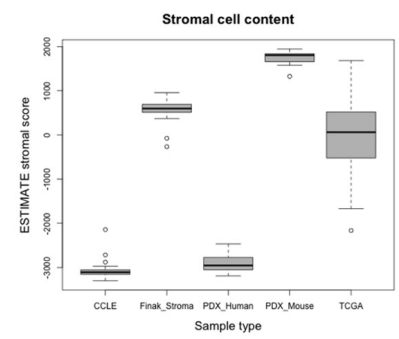

D

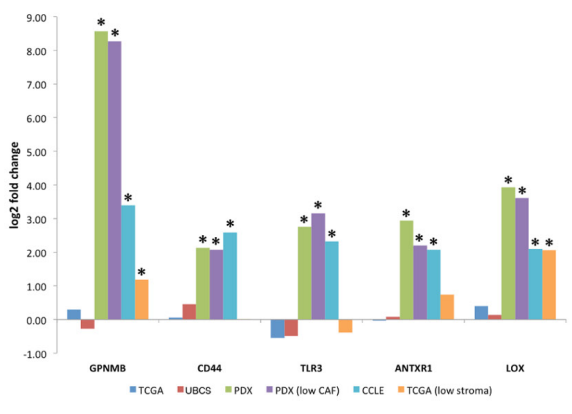

B

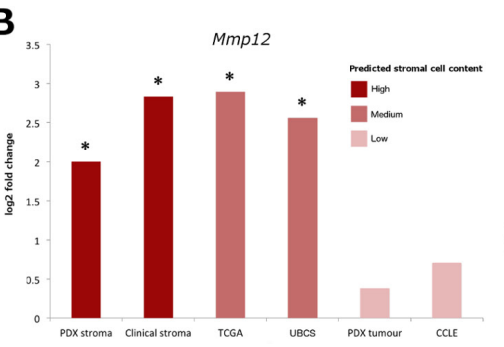

E

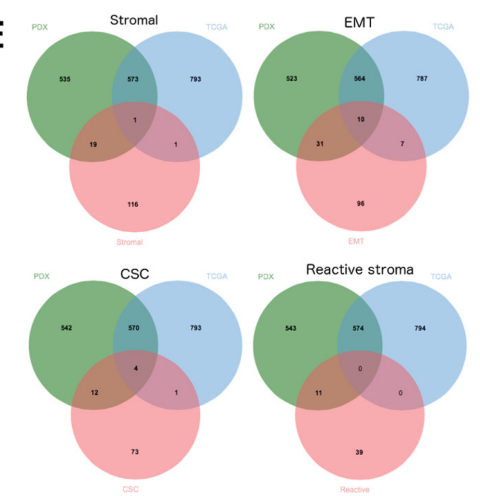

C

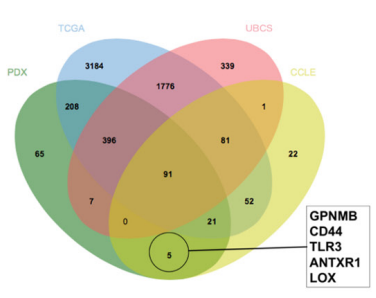

Figure 3: Compartment-specific gene expression markers of BTNBC. (A) comparison of ESTIMATE stromal score between PDX, TCGA, CCLE and clinical stroma samples. (B) fold changes achieved by MMP12 between BTNBC and ER+ luminal-B samples across all platforms $(* p<0.01)$. (C) derivation of tumor specific BTNBC markers exclusive to cell line and PDX datasets. (D) emergence of tumor specific markers in low stroma TCGA samples $\left({ }^{*} p<0.05\right)$. (E) overlap of stromal [10], EMT [20], CSC [21] and reactive stroma [22] signatures with BTNBC markers derived from human PDX and TCGA samples. (F) comparison of $\log _{10} p$-values achieved across sample types by overlap with each of the four signatures $\left({ }^{*} p<0.05\right)$. CSC: cancer stem cell, EMT: epithelial-mesenchymal transition, $M M P 12$ : Matrix Metalloproteinase 12. 
Table 2: Overlap of gene signatures with genes over-expressed in BTNBC and ER+ luminal-B breast cancers

BTNBC

\begin{tabular}{cccc}
\hline PDX & PDX FAP/ & TCGA & TCGA low \\
$\left(1127^{\mathrm{a}}\right)$ & CSPG4 & stroma \\
& low $\left(\mathbf{7 9 3}^{\mathrm{a}}\right)$ & $\left(\mathbf{1 3 6 8}^{\mathrm{a}}\right)$ & $\left(1763^{\mathrm{a}}\right)$ \\
\hline
\end{tabular}

ER+ luminal-B

$\begin{array}{cc}\text { PDX } & \text { PDX FAP/ } \\ \left(511^{\text {a }}\right) & \text { CSPG4 low } \\ & \left(569^{\text {a }}\right)\end{array}$

TCGA

$\left(876^{\mathrm{a}}\right)$

TCGA low

stroma

$\left(847^{\mathrm{a}}\right)$

\begin{tabular}{|l|c|c|c|c|c|c|c|c|}
\hline Stromal $\left(137^{\mathrm{b}}\right)$ & $20^{*}$ & $14^{*}$ & 2 & 9 & 1 & 5 & 4 & 6 \\
\hline CSC Up $\left(90^{\mathrm{c}}\right)$ & $16^{*}$ & $11^{*}$ & 5 & $16^{*}$ & 2 & 3 & 1 & 1 \\
\hline CSC Down $\left(211^{\mathrm{c}}\right)$ & 14 & 12 & 13 & 18 & $15^{*}$ & $14^{*}$ & $27^{*}$ & $29^{*}$ \\
\hline EMT Up $\left(144^{\mathrm{d}}\right)$ & $41^{*}$ & $27^{*}$ & 17 & $37^{*}$ & 5 & $11^{*}$ & 8 & 11 \\
\hline EM Down $\left(156^{\mathrm{d}}\right)$ & $30^{*}$ & $22^{*}$ & $34^{*}$ & $33^{*}$ & 8 & 6 & $21^{*}$ & $21^{*}$ \\
\hline $\begin{array}{l}\text { Reactive stroma } \\
(50)\end{array}$ & $11^{*}$ & $8^{*}$ & 0 & 6 & 0 & 0 & 1 & 1 \\
\hline
\end{tabular}

${ }^{*} p<0.05$ by hyper-geometric test.

${ }^{a}$ Number of genes over-expressed in TNBC or ER+ luminal-B.

${ }^{b}$ From [10].

'Breast cancer stem cell (CSC) signature from [21].

${ }^{\mathrm{d} E p i t h e l i a l-m e s e n c h y m a l ~ t r a n s i t i o n ~(E M T) ~ s i g n a t u r e ~ f r o m ~[20] . ~}$

${ }^{\mathrm{e}}$ From [22].

and mouse component. This approach identified known associations between tumor epithelial cell type and fibroblast activation [13], exemplified by significant correlation between human epithelial and mouse CAF markers. These were amongst several mouse genes correlated with human $\mathrm{CDH1}$ including numerous endothelial-associated genes. We then applied more stringent criteria to generate a crossspecies correlation network and identify novel associations. The strongest relationship was an anti-correlation between human MIF and mouse $D d x 6$. MIF plays an important role in the induction of vascular endothelial growth factor (VEGF) activity promoting blood vessel growth [27], and is also a target of the transcription factor hypoxiainducible factor 1-alpha (HIF1 $\alpha)$ [14]. Conversely, DDX6 inhibits VEGF protein expression under normoxia through binding to the $5^{\prime}$-UTR of its mRNA [15]. Our data suggest a potential stromal role for $D D X 6$ in the hypoxia response as an alternative to its previously established tumor-specific activity, and a potential link with $M I F$ activity in the tumor.

Overall, our findings highlight the value of RNASeq as a sensitive approach to dissect the complex interplay between both species compartments. However there are two caveats. Firstly the original tumor-stroma interactions in the patient are unlikely to be completely recapitulated due to incompatibility of human and mouse proteins, and the use of immune-compromised mice to promote xenograft establishment. Whether this is major caveat remains to be established. Whilst our results suggest known interactions develop in PDX models, further work will be necessary to establish a direct functional link in any novel interaction suggested by these data alone, and whether it represents a clinically relevant association or one that is unique to the xenograft. Secondly, the majority of tumors were implanted subcutaneously providing an ectopic environment. Where technically achievable, comparison with a more physiological implantation site used in orthotopic engraftment may better recapitulate the original patient stroma, and as such models become more widely available, similar profiling studies should be performed on these.

\section{Stromal gene expression markers of cancer- subtype}

Compared to the transcriptional differences observed between lung and breast cancer subtypes within the human tumor component, typically involving $>1000$ genes, the differences between the mouse stroma were small. Indeed, no mouse genes were deemed differentially expressed between lung adenocarcinoma and squamous mouse stroma, and only 18 mouse genes between breast ER+ and TN. This suggests recruitment of mouse stroma to the tumor is largely independent of disease subtype.

The search for stromal specific markers of BTNBC also highlighted potential disparities between PDX mouse and patient stroma. Whilst there was consensus in terms 
of fewer changes in stroma compared to tumor, only $M M P 12$, previously associated with breast cancer poor prognosis [17] [28], was detected as a BTNBC marker in both PDX mouse and patient stroma. The patient stroma clearly reflected changes typically seen in the tumor between TNBC and ER+ samples in agreement with recent studies suggesting that intrinsic breast cancer subtypes can be recapitulated based on the transcriptome of cancer adjacent tissue [29-31]. By contrast, there was no evidence of subtype reflected in the PDX stroma transcriptome but instead genes differentially expressed in PDX stroma were enriched in functions characteristic of metastasis, a process common to BTNBC.

Whilst these results could reflect intrinsic differences between the patient and mouse stroma, alternatively they could be a consequence of the higher predicted stromal cell purity achieved with our RNA-Seq approach compared to laser capture micro-dissection. Therefore, the specificity achieved by RNA-Seq in disambiguating human from mouse could offer a significant advantage, particularly in combination with a systematic integration of clinical datasets such as TCGA to overcome limitations of using mouse stroma. For example, evidence for MMP12 as a BTNBC stromal marker was strengthened by the observation that its expression is absent in samples containing only tumor cells, but present in samples that typically contain a significant stromal cell component.

\section{Expression signals from recruited stroma could mask presence of poor prognosis markers in BTNBC}

A major outcome of the disease-specific analysis was the identification of a BTNBC stromal signature in the PDX human component, not detected in TCGA or UBCS clinical samples. This was achieved through the ability of RNA-Seq to differentiate the two major sources of cells with a stromal phenotype in a typical xenograft sample, trans-differentiated tumor cells [32] (human cells) and coopted/recruited stroma (mouse cells). In a patient sample, this is difficult to accomplish with high confidence, even with specialist techniques such as laser capture microdissection, highlighting the capacity of RNA-Seq to refine complex signatures that are typically derived from heterogeneous cell populations.

The enrichment of breast CSC markers, including CD44, and EMT markers such as VIM supports previous work that showed EMT and CSC phenotypes are more likely to occur in basal breast cancers [33-34]. Notably, CD44 was one of five genes previously associated with BTNBC and poor prognosis that were clearly overexpressed in BTNBC PDX human and cell line samples but not in clinical samples. Of the other four genes, TLR3 and $A N T X R 1$ are also CSC markers [35-36], whilst $L O X$ and GPNMB are metastatic markers of BTNBC [37-40]. Given the phenotypes associated with these genes, we could have uncovered a particularly aggressive sub-population of cells analogous to CSCs residual after conventional treatments [21] that are present in BTNBC but absent in ER+ luminal-B patients. Critically, the observation that CSC and EMT gene signatures emerge in BTNBC markers derived from a dataset restricted to TCGA samples with the lowest stromal cell content strongly suggests that the phenomenon is not exclusive to PDX models, and counters the possibility that CSCs are simply more successful at engrafting in the mouse mammary microenvironment than other cell types [41].

We had two further concerns about our PDX breast cancer dataset, firstly BTNBC is over-represented due to the difficulty in establishing ER+ tumors in mice [42], and secondly, despite the retention of poor prognosis markers after removal of five BTNBC PDX models expressing CAF markers, we could not completely rule out the possibility of persistent patient stroma retention confounding the above observations. If this is the case, the presence of stromal-associated genes in the BTNBC PDX human component could reflect the level of reactive stroma in the original patient tumor. This indicates increased metastatic capacity leading to disease progression and poor prognosis, particularly in BTNBC [43]. Recently, a study has shown that increased stroma has the opposite effect in ER+ breast tumors [44], and although the results have since been challenged [45], a bias towards aggressive tumors with poor prognosis in PDX models could lead to an enrichment of high stroma BTNBC and low stroma $\mathrm{ER}+$ luminal-B tumors in our breast cancer cohort and the appearance of stromal-associated genes not present in the clinical data. Indeed, ER+ luminal-B tumors are themselves distinct from other ER+ subtypes due to their aggressive phenotype and resistance to conventional hormone therapy [46]. Therefore, the effect of excluding mouse stroma is to reveal genes specific to the original reactive stroma in the patient.

One further possibility was we had simply identified the presence of a stromal-like BTNBC subtype, however we found no evidence of bias towards mesenchymal or MSL TNBC subtypes [23] in our breast xenografts, nor in our TCGA/UBCS clinical samples of high tumor purity in which the signature emerged.

Despite these concerns, the masking of key markers of BTNBC by expression signals from recruited stroma is a significant observation and clearly illustrates the confounding effects of tumor sample heterogeneity on differential expression measurements. Considering the importance of the genes missed in the clinical samples unless stromal recruitment is accounted for, our observations have implications for any study that uses clinical samples to derive cancer-associated signatures, and potentially contribute to the poor overlap observed between signatures derived from different studies. They also demonstrate the potential of the RNA-Seq approach to refine complex signatures whose source compartment is ambiguous. 


\section{CONCLUSIONS}

This study has established RNA-Seq as a costeffective approach to enable simultaneous analysis of human tumor and mouse stroma across a pan-tumor explant panel. The analytical strategy provided new insights into mouse stromal heterogeneity and compartmentspecific disease markers otherwise overlooked by other technologies. Whilst further work is necessary to investigate the clinical relevance of the findings, they highlight the use of the technology as a platform to explore mouse stroma recruitment to the human tumor, and, critically, how this may influence therapeutic response. The strategy can also be applied routinely in pharmacodynamic studies of PDX models for detailed monitoring of compartment specific changes after treatment, ultimately leading to better efficacy prediction in the patient. As a resource for the pre-clinical model research community, all gene-level human and mouse expression data have been deposited in the ArrayExpress database (www.ebi.ac.uk/arrayexpress) under accession number E-MTAB-3980.

\section{METHODS}

\section{Ethics statement}

All animal studies were conducted in accordance with U.K. Home Office legislation, the Animal Scientific Procedures Act 1986, as well as the AstraZeneca Global Bioethics policy. All experimental work is outlined in project license 40/3483, which has gone through the AstraZeneca Ethical Review Process. Studies in the United States were conducted in accordance with the guidelines established by the internal IACUC (Institutional Animal Care and Use Committee) and reported following the ARRIVE (Animal Research: Reporting in vivo experiments) guidelines.

\section{Animals}

Female C.B.-17 severe combined immunodeficient (SCID) mice were purchased from Charles River Laboratories (Wilmington, MA). NOD/SCID/IL2R $\gamma$ null (NSG) mice were purchased from Jackson Laboratories (Bar Harbor, ME). Beige Nude XID mice were purchased from Harlan Laboratories (Madison, WI). Mice were housed under pathogen-free conditions in individual ventilated cages (IVC) at our Association for the Assessment and Accreditation of Laboratory Animal Care (AAALAC) accredited facility in Waltham, MA. All animal manipulations were conducted in a biosafety cabinet maintained under positive pressure.

\section{PDX model establishment}

In-house PDX models were established from fresh patient tissue procured from Maine Medical Center
BioBank (Portland, ME) and consented according to the Human Biological Samples Policy. Samples were received within 24 hours of surgery, minced and implanted subcutaneously into either NSG or CB17 SCID mice. The HBXF-079 sample was implanted orthotopically. The CTC-174 model was developed from circulating tumor cells in patient blood samples procured from Conversant Biologics (Huntsville, AL) and consented according to the Human Biological Samples Policy. The cells were isolated and implanted into the mammary fat pad of NSG mice. In addition, several models were purchased from Jackson Laboratories (Sacramento, CA) as tumor-bearing NSG mice. In most cases, the models were serially transplanted into fresh NSG mice as fragments for expansion and characterization, except BR0869F. For the remaining models, small pieces of PDX derived tumor were purchased specifically for profiling from Experimental Pharmacology and Oncology (EPO; http://www.epoberlin.com), Xentech (http://www.xentech.eu) and Champions (http://championsoncology.com) representing athymic nude, SCID, or NSG mice (Table S1).

\section{RNA extraction}

$\sim 50 \mathrm{mg}$ of tissue were cut from the frozen tumors and RNA isolated using the Qiazol kit with a DNase digestion using the RNase-free DNase Kit (Qiagen) on the Qiacube according to manufacturer's instructions. RNA concentration was measured using the NanoDrop ND1000 (NanoDrop), and quality determined using the Agilent RNA nano 6000 kit and Bioanalyzer (Agilent Technologies). RNA integrity numbers (RIN) for all samples fell between 7 and 10 .

\section{RNA-Seq data}

RNA libraries were made with the Illumina TruSeq RNA Sample Preparation kit (un-stranded) according to the manufacturer's protocol. These libraries were then submitted for 100 bp paired-end sequencing on the Illumina HiSeq 2000 platform using one lane per three to six PDX models. A concatenated human (GRCh37/hg19) and mouse (GRCm38/mm9) genome was then constructed to form a single genome of 43 chromosomes (23 from human and 20 from mouse). This was indexed using StarAlign [48] and a "gtf" formatted file combining annotations from both human and mouse genes downloaded from Ensembl version 75 [47]. The length of donor/acceptor sequence ("sjdbOverhang") either side of a splice junction was set to 99 bases, with all other parameters set to their defaults. The sequenced reads were aligned to the human-mouse genome using StarAlign [48] with no more than three mismatches across each end of the pair allowed, and reads mapped to multiple locations discarded. Reads whose ratio of mismatches to mapped length was greater than 0.10, and non-canonical splice junctions were also removed. All other parameters were set to their defaults for non-stranded 
alignment. Since we only considered reads uniquely mapped to the human-mouse genome according to the mapping parameters above, reads mapping to both human and mouse genomes were automatically discarded. Therefore, the output of the pipeline was a set of species-specific reads that mapped uniquely either to the human or mouse genome.

\section{Measurement of expression level}

The number of reads overlapping each gene present in both human (GRCh37) and mouse (GRCm38) annotation files downloaded from Ensembl version 75 [47] were calculated using the R Bioconductor package HTSeq [49] in un-stranded, union mode with all other parameters set to default values. For the same group of genes, expression based on Fragments Per Kilobase per Million fragments mapped (FPKM) was estimated using Cuffnorm with library type defined as "fr-unstranded" and all other parameters set to defaults [50]. Non-protein coding genes were ignored, as well as genes whose largest transcript is less that $400 \mathrm{bp}$ due to potential over-estimation of expression across transcripts less than the average fragment length. For PDX model BR0555 represented by two biological and three technical replicates, the replicate whose mouse component achieved the highest ESTIMATE stromal score was chosen for downstream analyses. Where required, mouse genes names were converted to human and vice versa using MammalHom (http://depts. washington.edu/l21/mammalhom.html).

\section{Clustering of gene expression data with consensus non-negative matrix factorization (NMF)}

We applied NMF to cluster the human and mouse transcriptomes and identify tumor and stromal specific subtypes respectively. The underlying principle of NMF is dimensionality reduction in which a small number of meta-genes, each defined as a positive linear combination of the genes in the expression data, are identified and then used to group samples into clusters based on the gene expression pattern of the samples as positive linear combinations of these meta-genes. Using the $\mathrm{R}$ package $N M F$ [51], factorization rank $k$ was chosen by computing the clustering for $k=2-11$ against 50 random initializations of both the actual and a permuted gene expression matrix, and selecting the $k$ value achieving the largest difference between cophenetic correlation coefficients calculated from the actual and permutated data (Figure S8). For further visual confirmation of a sensible choice of $k$, consensus matrices were generated corresponding to different $k$ values (Figure S9). To achieve stability, the NMF algorithm was then run against 200 perturbations of each gene expression matrix at the chosen values of $k=9$ (human) and $k=5$ (mouse).

\section{Breast cancer differential expression analyses}

\section{PDX models}

PDX model hormone receptor status was determined by immunohistochemistry, and broadly correlated with mRNA expression. Nine of the 10 TNBC PDX samples were basal-like and all four ER+ PDX samples luminal-B according to PAM50 classification [52] (Table S10) therefore comparisons were restricted to these two intrinsic subtypes. TNBC subtypes listed in Table S11A were predicted using TNBCtype [53] with FPKM expression values as input. Two tumors (HBCX7 and HBCX19) that had undergone metastasis were ignored. Differentially expressed genes were identified using the R Bioconductor package DESeq2 [54]. "minReplicatesForReplace" was set to seven or size of the smallest group if one group contained less than seven samples. All other parameters were set as defaults. Only genes achieving FPKM $>1$ in at least one sample were input to DESeq2. Differential expressed genes were defined as those achieving a $\left|\log _{2} \mathrm{FC}\right|>1.5$ and FDR $<0.05$.

\section{Patient stroma}

Agilent-012391 Whole Human Genome Oligo Microarray G4112A expression data for a set of 45 human breast cancer samples whose stroma had been isolated from the tumor by laser micro-dissection [17] were downloaded from the Gene Expression Omnibus [55] (GSE9014). Raw expression data in the Agilent Feature Extraction text files were loess normalized and differential expression between seven TNBC and $38 \mathrm{ER}+$ samples calculated using the R Bioconductor package Limma [56]. Probesets achieving a $\left|\log _{2} \mathrm{FC}\right|>1.5$ and FDR $<0.05$ were deemed differentially expressed.

\section{UBCS}

Fresh frozen breast tissue samples were obtained from 88 women who had surgery at the Huntsman Cancer Hospital from 2009-2012, including tumor tissues from 69 breast cancer patients. One tumor sample yielded poor quality RNA $(\mathrm{RIN}=2.5)$ and was removed from consideration, resulting in a panel of 68 tumor samples. RNA libraries were made with the Illumina TruSeq Stranded mRNA Sample Preparation kit with oligo dT selection according to the manufacturer's protocol. These libraries were then submitted for $50 \mathrm{bp}$ single-end sequencing on the Illumina HiSeq 2000 platform using eight samples per lane. The resulting reads were aligned to the human (GRCh37/hg19) genome using StarAlign [48] with no more than three mismatches and only uniquely mapped reads allowed. Reads whose ratio of mismatches to mapped length was greater than 0.10 were also discarded. All other parameters were set to their defaults for stranded alignment. The number of reads overlapping each gene 
present in the human (GRCh37) annotation file downloaded from Ensembl version 75 [47] were calculated using the R Bioconductor package HTSeq [49] in stranded, union mode with all other parameters set to default values. The resulting gene-by-sample matrix consisted of $12 \mathrm{ER}+$ luminal B and 10 BTNBC samples. TNBC subtypes listed in Table S11B were predicted using TNBCtype [53] with FPKM expression values as input. Differentially expressed genes $\left(\left|\log _{2} \mathrm{FC}\right|>1.5\right.$ and FDR $\left.<0.05\right)$ were identified with DESeq2 [54] using the same protocol applied to the PDX models.

\section{TCGA}

TCGA gene expression data (June 2014) processed using the RNASeqv2 pipeline were downloaded from the Broad Institute GDAC Firehose (http://gdac.broadinstitute. org) and parsed to generate a gene-by-sample matrix of raw counts across solid tumor samples only. Breast cancer samples treated with tamoxifen were discarded as were genes achieving a mean count $<1$ across all remaining samples. The resulting matrix consisted of 18,488 genes across 79 luminal B ER+ and 54 TNBC samples as classified by TCGA Network [19] by PAM50 [52]. TNBC subtypes given in Table S11C were taken from [56]. Differential expression was calculated using DESeq2 [54] as for the PDX models.

\section{CCLE}

RMA normalized Affymetrix U133+2 expression data was downloaded from CCLE [18], and all non-breast cancer cell lines were removed as well as cell lines for which subtype was ambiguous according to [58]. Since no PDX or TCGA basal-like samples were classified as claudin-low, these were removed from the cell line cohort. The resulting gene-by-sample matrix consisted of $12 \mathrm{ER}+$ luminal B and seven BTNBC samples. TNBC subtypes given in Table S11D were taken from [23]. Genes achieving $|\log 2 \mathrm{FC}|>1.5$ and $T$-test $p$-value $<0.01$ were defined as differentially expressed.

Note that for cross-platform comparisons, we considered only a core set of 15,984 genes represented on all platforms.

\section{Expression data preparation for clustering and cross-species correlation analysis}

Gene-by-sample expression matrices were generated for each species in which only genes achieving FPKM $>10$ (human genes) or FPKM $>2$ (mouse genes) in at least one sample, and coefficient of variation $>0.20$ were retained. The resulting matrix of FPKM expression values was converted to a non-negative matrix by addition of an arbitrary value of 1.1 to each entry and then taking $\log _{2}$ of the result.

\section{EMT and breast CSC signatures}

The EMT signature was taken from [20] consisting of 144 human (141 mouse) genes up-regulated and 156 human (142 mouse) genes down-regulated in EMT according to [20]. The breast CSC signature was based on the $\mathrm{CD}_{4}{ }^{+} /$ $\mathrm{CD} 24^{-/ \text {low }}$-mammosphere (MS) signature of [21] using 90 up-regulated and 211 down-regulated human genes.

\section{Mouse gender predictions}

All genes on the Y chromosome were identified, and pseudogenes and genes achieving a count of $<1000$ reads across all models were removed. The remaining genes were manually inspected, and those with evidence of expression across all models and therefore not following the profile of the majority of genes were also removed. Genes contributing to the mouse gender prediction consisted of Eif $2 s 3 y, D d x 3 y, K d m 5 d$ and Uty. Read counts across these genes were summed for all models and the results divided by the total read count across all genes and multiplied by 1000 to give a Y index. Mice achieving $\mathrm{Y}$ index $>10$ were classified as male, and female otherwise.

\section{ACKNOWLEDGMENTS}

The authors would like to thank Beijing Genomics Institute for performing the sequencing, Sara Dempster (AstraZeneca) for initiating the bioinformatics, Corinne Reimer for input to model development, Brian Dougherty for help in coordinating model sequencing, Guoying Wang for technical help in generating the UBCS data, and Brian Dalley of the High Throughput Genomics Core (University of Utah) for UBSC RNA sequencing. Thanks are also extended to Dr Will English for insightful discussions, and Professor Angela Cox for critical reading of the manuscript.

\section{GRANT SUPPORT}

All research was funded by AstraZeneca, except the UBCS data, which was funded by the National Cancer Institute (R01 CA163353), the Avon Foundation (022009-080), and the Komen Foundation (BCTR0706911), and supported by the Biospecimen and Pathology Core that is partially funded by P30 CA42014 Comprehensive Cancer Center grant.

\section{CONFLICTS OF INTEREST}

All authors except NJC have either a current or past affiliation to the commercial funders of this research (AstraZeneca). 


\section{REFERENCES}

1. Hanahan D, Weinberg RA. Hallmarks of Cancer: The Next Generation. Cell. 2011; 144:646-674. doi: 10.1016/j. cell.2011.02.013.

2. de Ridder D, van der Linden CE, Schonewille T, Dik WA, Reinders MJ, van Dongen JJ, Staal FJ. Purity for clarity: the need for purification of tumor cells in DNA microarray studies. Leukemia. 2005; 19:618-627.

3. Tentler JJ, Tan AC, Weekes CD, Jimeno A, Leong S, Pitts TM, Arcaroli JJ, Messersmith WA, Eckhardt SG. Patient-derived tumour xenografts as models for oncology drug development. Nat Rev Clin Oncol. 2012; 9:338-350. doi: 10.1038/ nrclinonc.2012.61.

4. Hidalgo M, Amant F, Biankin, AV, Budinská E, Byrne AT, Caldas C, Clarke RB, de Jong S, Jonkers J, Mælandsmo GM, Roman-Roman S, Seoane J, Trusolino L, et al. Patient-derived xenograft models: an emerging platform for translational cancer research. Cancer Discovery. 2014; 4:998-1013. doi: 10.1158/2159-8290.CD-14-0001.

5. Guilhamon P, Butcher LM, Presneau N, Wilson GA, Feber A, Paul DS, Schütte M, Haybaeck J, Keilholz U, Hoffman J, Ross MT, Flanagan AM, Beck S. Assessment of patientderived tumour xenografts (PDXs) as a discovery tool for cancer epigenomics. Genome Medicine. 2014, 6:116. doi: 10.1186/s13073-014-0116-0.

6. Bradford JR, Farren M, Powell SJ, Runswick S, Weston SL, Brown H, Delpuech O, Wappett M, Smith NR, Carr TH, Dry JR, Gibson NJ, Barry ST. RNA-Seq differentiates tumour and host mRNA expression changes induced by treatment of human tumour xenografts with the VEGFR tyrosine kinase inhibitor cediranib. PLOS ONE. 2013; 8:66003. doi: 10.1371/ journal.pone.0066003.

7. Conway T, Wazny J, Bromage A, Tymms M, Sooraj D, Williams ED, and Beresford-Smith B. Xenome - a tool for classifying reads from xenograft samples. Bioinformatics. 2012; 28:172-178. doi: 10.1093/bioinformatics/bts236.

8. Isella C, Terrasi A, Bellomo SE, Petti C, Galatola G, Muratore A, Mellano A, Senetta R, Cassenti A, Sonetto C, Inghirami G, Trusolino L, Fekete $\mathrm{Z}$, et al. Stromal contribution to the colorectal cancer transcriptome. Nature Genetics. 2015; 47:312-319. doi: 10.1038/ng.3224.

9. Raskatov JA, Nickols NG, Hargrove AE, Marinov GK, Wold B, Dervan PB. Gene expression changes in a tumor xenograft by a pyrrole-imidazole polyamide. Proc Natl Acad Sci U S A. 2012; 109:16041-16045. doi:10.1073/ pnas. 1214267109.

10. Yoshihara K, Shahmoradgoli M, MartínezE, Vegesna R, KimH, Torres-Garcia W, Treviño V, Shen H, Laird PW, Levine DA, Carter SL, Getz G, Stemke-Hale K, et al. Inferring tumour purity and stromal and immune cell admixture from expression data. Nature Comms. 2013; 4: 2612. doi:10.1038/ ncomms3612.

11. Chen J, Bardes EE, Aronow BJ, Jegga AG. ToppGene Suite for gene list enrichment analysis and candidate gene prioritization. Nucleic Acids Res. 2009; 37: W305-311. doi: 10.1093/nar/gkp427.

12. Desmedt C, Majjaj S, Kheddoumi N, Singhal SK, HaibeKains B, El Ouriaghli F, Chaboteaux C, Michiels S, Lallemand F, Journe F, Duvillier H, Loi S, Quackenbush J, et al. Characterization and clinical evaluation of CD10+ stroma cells in the breast cancer microenvironment. Clin Cancer Res. 2012; 18:1004-1014. doi: 10.1158/1078-0432.CCR-11-0383.

13. Eberlein C, Rooney C, Ross SJ, Farren M, Weir HM, Barry ST. E-Cadherin and EpCAM expression by NSCLC tumour cells associate with normal fibroblast activation through a pathway initiated by integrin $\alpha \mathrm{v} \beta 6$ and maintained through TGF $\beta$ signalling. Oncogene. 2015; 34:704-716. doi: 10.1038/ onc.2013.600.

14. Baugh JA, Gantier M, Li L, ByrneA, Buckley A, Donnelly SC. Dual regulation of macrophage migration inhibitory factor (MIF) expression in hypoxia by CREB and HIF-1. Biochem Biophys Res Commun. 2006; 347:895-903.

15. de Vries S, Naarmann-de Vries IS, Urlaub H, Lue H, Bernhagen J, Ostareck DH, Ostareck-Lederer A. Identification of DEAD-box RNA helicase 6 (DDX6) as a cellular modulator of vascular endothelial growth factor expression under hypoxia. J Biol Chem. 2013; 288:5815-5827. doi: 10.1074/jbc.M112.420711.

16. Winter SC, Buffa FM, Silva P, Miller C, Valentine HR, Turley H, Shah KA, Cox GJ, Corbridge RJ, Homer JJ, Musgrove B, Slevin N, Sloan P, et al. Relation of a hypoxia metagene derived from head and neck cancer to prognosis of multiple cancers. Cancer Res. 2007, 67:3441-3449.

17. Finak G, Bertos N, Pepin F, Sadekova S, Souleimanova M, Zhao H, Chen H, Omeroglu G, Meterissian S, Omeroglu A, Hallett M, Park M. Stromal gene expression predicts clinical outcome in breast cancer. Nat Med. 2008; 14: 518527. doi: $10.1038 / \mathrm{nm} 1764$.

18. Barretina J, Caponigro G, Stransky N, Venkatesan K, Margolin AA, Kim S, Wilson CJ, Lehár J, Kryukov GV, Sonkin D, Reddy A, Liu M, Murray L, et al. The Cancer Cell Line Encyclopedia enables predictive modelling of anticancer drug sensitivity. Nature. 2012; 483:603-607. doi: 10.1038/nature11003.

19. The Cancer Genome Atlas Network. Comprehensive molecular portraits of human breast tumours. Nature. 2012; 490:61-70. doi: 10.1038/nature11412.

20. Loboda A, Nebozhyn MV, Watters JW, Buser CA, Shaw PM, Huang PS, Van't Veer L, Tollenaar RA, Jackson DB, Agrawal D, Dai H, Yeatman TJ. EMT is the dominant program in human colon cancer. BMC Med Genomics. 2011; 4:9. doi: 10.1186/1755-8794-4-9.

21. Creighton CJ, Li X, Landis M, Dixon JM, Neumeister VM, Sjolund A, Rimm DL, Wong H, Rodriguez A, Herschkowitz JI, Fan C, Zhang X, He X, et al. Residual breast cancers after conventional therapy display mesenchymal as well as tumor-initiating features. Proc Natl Acad Sci USA. 2009; 106:13820-13825. doi: 10.1073/pnas.0905718106. 
22. Farmer $\mathrm{P}$, Bonnefoi H, Anderle $\mathrm{P}$, Cameron D, Wirapati $\mathrm{P}$, Becette V, André S, Piccart M, Campone M, Brain E, Macgrogan G, Petit T, Jassem J, et al. A stroma-related gene signature predicts resistance to neoadjuvant chemotherapy in breast cancer. Nat Med. 2009; 15:68-74. doi: 10.1038/ nm.1908.

23. Lehmann $\mathrm{BD}$, Bauer JA, Chen $\mathrm{X}$, Sanders ME, Chakravarthy AB, Shyr Y, Pietenpol JA. Identification of human triple-negative breast cancer subtypes and preclinical models for selection of targeted therapies. J Clin Invest. 2011; 121:2750-2767. doi: 10.1172/JCI45014.

24. Prat A, Adamo B, Cheang MCU, Anders CK, Carey LA, Perou CM. Molecular Characterization of Basal-Like and Non-Basal-Like Triple-Negative Breast Cancer. Oncologist. 2013; 18:123-133. doi: 10.1634/theoncologist.2012-0397.

25. Hylander BL, Punt N, Tang H, Hillman J, Vaughan M, Bshara W, Pitoniak R, and Repasky EA. Origin of the vasculature supporting growth of primary patient tumor xenografts. J Transl Med. 2013; 11:110. doi: 10.1186/14795876-11-110.

26. Garrido-Laguna I, Uson M, Rajeshkumar NV, Tan AC, de Oliveira E, Karikari C, Villaroel MC, Salomon A, Taylor G, Sharma R, Hruban RH, Maitra A, Laheru D, et al. Tumor engraftment in nude mice and enrichment in stroma- related gene pathways predict poor survival and resistance to gemcitabine in patients with pancreatic cancer. Clin Cancer Res. 2011, 17:5793-5800. doi: 10.1158/1078-0432.CCR11-0341.

27. Winner M, Koong AC, Rendon BE, Zundel W, Mitchell RA. Amplification of tumor hypoxic responses by macrophage migration inhibitory factor-dependent hypoxia-inducible factor stabilization. Cancer Res. 2007, 67:186-193.

28. McGowan PM, Duffy MJ. Matrix metalloproteinase expression and outcome in patients with breast cancer: analysis of a published database. Ann Oncol. 2008; 19:1566-1572. doi: 10.1093/annonc/mdn180.

29. Roman-Perez E, Casbas-Hernandez P, Pirone J, Rein J, Carey L, Lubet R, Mani SA, Amos KD, Troester MA. Gene expression in extra-tumoral microenvironment predicts clinical outcome in breast cancer patients. Breast Cancer Res. 2011; 14:R51.

30. Graham K, Ge X, de las Morenas A, Tripathi A, and Rosenberg CL. Gene expression profiles of estrogen receptor positive and estrogen receptor negative breast cancers are detectable in histologically normal breast epithelium. Clin Cancer Res. 2011, 17:236-246. doi: 10.1158/1078-0432. CCR-10-1369.

31. Casbas-Hernandez P, Sun X, Roman-Perez E, D'Arcy M, Sandhu R, Hishida A, McNaughton KK, Yang XR, Makowski L, Sherman ME, Figueroa JD, Troester MA. Tumor intrinsic subtype is reflected in cancer-adjacent tissue. Cancer Epidemiol Biomarkers Prev. 2015; 24: 406-414. doi: 10.1158/1055-9965.EPI-14-0934.

32. Shekhani MT, Jayanthy AS, Maddodi N, Setaluri V. Cancer stem cells and tumor transdifferentiation: implications for novel therapeutic strategies. Am J Stem Cells. 2013; 2:52-61.

33. Sarrió D, Rodriguez-Pinilla SM, Hardisson D, Cano A, Moreno-Bueno G, Palacios J. Epithelial-mesenchymal transition in breast cancer relates to the basal-like phenotype. Cancer Res. 2008; 68:989-997. doi: 10.1158/0008-5472. CAN-07-2017.

34. Honeth G, Bendahl P-O, Ringnér M, Saal LH, GruvbergerSaal SK, Lövgren K, Grabau D, Fernö M, Borg A, Hegardt C. The CD44+/CD24- phenotype is enriched in basal-like breast tumors. Breast Cancer Res. 2008; 10:R53. doi: 10.1186/bcr2108.

35. Jia D, Yang W, Li L, Liu H, Tan Y, Ooi S, Chi L, Filion LG, Figeys D, Wang L. $\beta$-Catenin and NF- $\kappa \mathrm{B}$ co-activation triggered by TLR3 stimulation facilitates stem cell-like phenotypes in breast cancer. Cell Death Differ. 2015; 22:298-310. doi: 10.1038/cdd.2014.145.

36. Chen D, Bhat-Nakshatri P, Goswami C, Badve S, Nakshatri H. ANTXR1, a stem cell enriched functional biomarker, connects collagen signalling to cancer stem-like cells and metastasis in breast cancer. Cancer Research. 2013; 73:5821-5833. doi: 10.1158/0008-5472.CAN-13-1080.

37. Rose AA, Grosset AA, Dong Z, Russo C, Macdonald PA, Bertos NR, St-Pierre Y, Simantov R, Hallett M, Park M, Gaboury L, Siegel PM. Glycoprotein nonmetastatic B is an independent prognostic indicator of recurrence and a novel therapeutic target in breast cancer. Clin Cancer Res. 2010; 16:2147-2156. doi: 10.1158/1078-0432.CCR-09-1611.

38. Erler JT, Giaccia AJ Lysyl oxidase mediates hypoxic control of metastasis. Cancer Res. 2006; 66:10238-10241.

39. Erler JT, Bennewith KL, Cox TR, Lang G, Bird D, Koong A, Le QT, Giaccia AJ. Hypoxia-induced lysyl oxidase is a critical mediator of bone marrow cell recruitment to form the premetastatic niche. Cancer Cell. 2009; 15:35-44. doi: 10.1016/j.ccr.2008.11.012.

40. Chu IM, Michalowski AM, Hoenerhoff M, Szauter KM, Luger D, Sato M, Flanders K, Oshima A, Csiszar K, Green JE. GATA3 inhibits lysyl oxidase-mediated metastases of human basal triple-negative breast cancer cells. Oncogene. 2012; 31:2017-2027. doi: 10.1038/onc.2011.382.

41. Fillmore C, Kuperwasser C. Human breast cancer stem cell markers CD44 and CD24: enriching for cells with functional properties in mice or in man? Breast Cancer Res. 2007; 9:303.

42. Kabos P, Finlay-Schultz J, Li C, Kline E, Finlayson C, Wisell J, Manuel CA, Edgerton SM, Harrell JC, Elias A, Sartorius CA. Patient-derived luminal breast cancer xenografts retain hormone receptor heterogeneity and help define unique estrogen-dependent gene signatures. Breast Cancer Res. Treat. 2012; 135:415-432. doi: 10.1007/s10549-012-2164-8.

43. de KruijfEM, van Nes JG, van de Velde CJ, Putter H, Smit VT, Liefers GJ, Kuppen PJ, Tollenaar RA, Mesker WE. Tumor-stroma ratio in the primary tumor is a prognostic factor in early breast cancer patients, especially in triplenegative carcinoma patients. Breast Cancer Res Treat. 2011; 125:687-696. doi: 10.1007/s10549-010-0855-6. 
44. Downey CL, Simpkins SA, White J, Holliday DL, Jones JL, Jordan LB, Kulka J, Pollock S, Rajan SS, Thygesen HH, Hanby AM, Speirs V. The prognostic significance of tumour-stroma ratio in oestrogen receptor-positive breast cancer. British Journal of Cancer. 2014; 110:1744-1747. doi: 10.1038/bjc.2014.69.

45. Mesker WE, Dekker TJA, de Kruijf EM, Engels CC, van Pelt GW, Smit VT, Tollenaar RA. Comment on: The prognostic significance of tumour-stroma ratio in oestrogen receptor-positive breast cancer. British Journal of Cancer. 2015; 112:1832-1833. doi: 10.1038/bjc.2014.570.

46. Creighton CJ. The molecular profile of luminal B breast cancer. Biologics. 2012; 6:289-297. doi: 10.2147/BTT. S29923.

47. Flicek P, Amode MR, Barrell D, Beal K, Billis K, Brent S, Carvalho-Silva D, Clapham P, Coates G, Fitzgerald S, Gil L, et al. Ensembl 2014. Nucleic Acids Research. 2014; 42:D749-D755. doi: 10.1093/nar/gkt1196.

48. Dobin A, Davis CA, Schlesinger F, Drenkow J, Zaleski C, Jha S, Batut P, Chaisson M, Gingeras TR. STAR: ultrafast universal RNA-seq aligner. Bioinformatics. 2013; 29:15-21. doi: 10.1093/bioinformatics/bts635.

49. Anders S, Pyl PT, Huber W. HTSeq - A Python framework to work with high-throughput sequencing data. Bioinformatics. 2015; 31:166-169. doi: 10.1093/bioinformatics/btu638.

50. Trapnell C, Williams BA, Pertea G, Mortazavi A, Kwan G, van Baren MJ, Salzberg SL, Wold BJ, Pachter L. Transcript assembly and quantification by RNA-Seq reveals unannotated transcripts and isoform switching during cell differentiation. Nature Biotechnology. 2010; 28:511-515. doi: 10.1038/ nbt.1621.

51. Gaujoux R, Seoighe C. A flexible R package for nonnegative matrix factorization. BMC Bioinformatics. 2010; 11:367. doi: 10.1186/1471-2105-11-367.

52. Parker JS, Mullins M, Cheang MCU, Leung S, Voduc D, Vickery T, Davies S, Fauron C, He X, Hu Z, Quackenbush JF, Stijleman IJ, Palazzo J, et al. Supervised risk predictor of breast cancer based on intrinsic subtypes. J Clin Oncol. 2009; 27:1160-1167. doi: 10.1200/JCO.2008.18.1370.
53. Chen X, Li J, Gray WH, Lehmann BD, Bauer JA, Shyr Y, Pietenpol JA. TNBCtype: A Subtyping tool for triplenegative breast cancer. Cancer Inform. 2012; 11:147-156. doi: 10.4137/CIN.S9983.

54. Love MI, Huber W, Anders S. Moderated estimation of fold change and dispersion for RNA-seq data with DESeq2. Genome Biology 2014; 15:550.

55. Barrett T, Wilhite SE, Ledoux P, Evangelista C, Kim IF, Tomashevsky M, Marshall KA, Phillippy KH, Sherman PM, Holko M, Yefanov A, Lee H, Zhang N, et al. NCBI GEO: archive for functional genomics data sets - update. Nucleic Acids Res. 2013; 41:D991-995. doi: 10.1093/nar/gks1193.

56. Smyth GK. Limma: linear models for microarray data. In: Gentleman R, Carey V, Dudoit S, Irizarry R, Huber W, editors. Bioinformatics and computational biology solutions using $\mathrm{R}$ and Bioconductor. New York: Springer. 2005; 397-420.

57. Lehmann BD, Bauer JA, Schafer JM, Pendleton CS, Tang L, Johnson KC, Chen X, Balko JM, Gómez H, Arteaga CL, Mills GB, et al. PIK3CA mutations in androgen receptorpositive triple negative breast cancer confer sensitivity to the combination of PI3K and androgen receptor inhibitors. Breast Cancer Res. 2014; 16:406. doi: 10.1186/s13058-014-0406-X.

58. Prat A, Karginova O, Parker JS, Fan C, He X, Bixby L, Harrell JC, Roman E, Adamo B, Troester M, Perou CM. Characterization of cell lines derived from breast cancers and normal mammary tissues for the study of the intrinsic molecular subtypes. Breast Cancer Res Treat. 2013; 142:237-255. doi: 10.1007/s10549-013-2743-3.

59. Shannon P, Markiel A, Ozier O, Baliga NS, Wang JT, Ramage D, Amin N, Schwikowski B, Ideker T. Cytoscape: a software environment for integrated models of biomolecular interaction networks. Genome Research. 2003; 13:2498-2504. 